\title{
PREPARAÇÃO E CARACTERIZAÇÃO NANOCOMPÓSITOS COM NANOTUBOS DE CARBONO DISPERSOS EM SURFACTANTES
}

\section{NANOCOMPOSITS PREPARATION AND CHARACTERIZATION WITH CARBON NANOTUBES DISPERSED IN SURFACTANTS}

\author{
Rocha, V. V. ${ }^{1}$ e Ludvig, P. ${ }^{1}$ \\ ${ }^{1}$ Programa de Pós-Graduação em Engenharia Civil - Centro Federal de Educação \\ Tecnológica de Minas Gerais - CEFET-MG \\ E-mail: vrvanessavilela@gmail.com.br
}

article info

Article history:

Received 2017-09-04

Accepted 2017-11-20

Available online 2017-12-20

RESUMO: A dispersão efetiva dos NTCs para preparação de compósitos de cimento é crucial para o alcance de ganhos de resistência. O comportamento hidrofóbico desse nanomaterial faz com que ele se aglomere em presença de água o que leva a formação de grânulos. Pesquisas envolvendo incorporação de NTCs em pastas de cimento têm sendo desenvolvidas, e o presente trabalho realizou experimentos de análise de dispersão em meio aquoso e preparação e caracterização de pastas de cimento com NTCs. Como resultado, foi observado que os NTCs se mostram dispersos em meio aquoso na presença de aditivos plastificantes a base de lignosulfonato e policarboxilato. No entanto, as pastas de cimento preparadas por esse método de dispersão não apresentaram ganhos de resistência significativos, sugerindo pouca eficiência de dispersão para esse fim.

\begin{abstract}
The effective dispersion of CNTs for cement composites preparation is crucial for resistance gains achievement. The hydrophobic behavior of this nanomaterial causes its agglomeration in the presence of water which leads to the formation of clusters. Research involving CNTs incorporation into cement pastes have been developed, and the present work carried out dispersion analysis experiments in aqueous media and preparation and characterization of cement pastes with CNTs. As a result, it has been observed that CNTs are well dispersed in aqueous media in the presence of plasticizers based on lignosulfonate and polycarboxylate. However, the cement pastes prepared by this dispersion method did not present significant resistance gains, suggesting little dispersion efficiency for this purpose.
\end{abstract}

\section{INTRODUÇÃO}

A descoberta dos nanotubos de carbono (NTCs) foi feita pelo pesquisador japonês Sumio Iijima na década de 90 e, devido as propriedades mecânicas do material, principalmente pela sua resistência à tração e o seu módulo de elasticidade, chamou muita atenção na área da Nanotecnologia. MAKAR et al. (2005) afirmam que os NTCs são uma única forma de carbono que possui propriedades mecânicas, térmicas e eletrônicas desejáveis. Devido a tais características, eles têm sido alvo dos estudos mais importantes na área de 
nanotecnologia (MAKAR e BEAUDOIN, 2004) e altamente promissores na incorporação de materiais cimentíceos para formação de nanocompósitos. Entretanto, as propriedades hidrofóbicas desse nanomaterial fazem com que em presença da água os mesmos tendam a formar grânulos prejudicando a resistência dos compósitos.

MELO et al. (2011) afirmam que para a melhor dispersão, é necessário que os nanotubos sejam submetidos a um tratamento capaz de aumentar as propriedades dispersivas. De acordo com FILHO e FAGAN (2007) um dos tipos de tratamento, denominado funcionalização, acontece através de interações, que podem ser não covalentes (ligações fracas com os NTCs) e covalentes (interações fortes que geram grandes modificações nas propriedades dos NTCs).

Atualmente tem sido mais estudado dois métodos de dispersão: (i) envolvendo a dispersão prévia em surfactante com sonificação, e (ii) a tentativa de dispersar os NTCs nas partículas de cimento ou alguma outra partícula (PAULA, 2014). LIU et al. (2007) relataram que os NTCs funcionalizados foram dispersos em meio ao surfactante lignosulfonato e apresentaram efetiva dispersão em água, o que indica que as interações entre os NTCs foram superadas por meio do processo adotado. Já COLLINS et al. (2012) elaboram um trabalho comparando a dispersão física de NTCs por meio de surfactantes do tipo polinaftaleno sulfonado (PNS), policarboxilato e lignosufonato, com proporções de NTCs variando de $0,5 \%$ à $2 \%$ de NTCs em relação ao peso do cimento. Para taxas de $0,5 \%$ os autores observaram ganhos na resistência de $25 \%$ quando dispersos em policarboxilato. Entretanto, quando dispersos em lignosulfonato os resultados foram $30,1 \%$ inferiores para a mesma concentração de nanotubos. Dessa forma, os autores sugerem que a melhor dispersão do nanomaterial é em meio à policarboxilato. Além disso, eles observaram que a presença de NTCs afeta a trabalhabilidade dos compósitos no estado fresco.

O presente trabalho objetiva analisar a dispersão dos NTCs em meio a solução aquosa com aditivos a base de lignosulfonato e policarboxilato no granulômetro a laser da marca CILAS, e, em sequência, preparar e caracterizar nanocompósitos de cimento com a incorporação de teores de $0 \%, 0,15 \%$ e $0,30 \%$ e $0,50 \%$ de $\mathrm{NTCs}$, em relação ao peso do cimento, dispersos também em aditivos a base de lignosulfonato e policarboxilato.

\section{METODOLOGIA}

\subsection{Materiais}

Para o desenvolvimento desse trabalho foram adotados quatro tipos de surfactante, dois a base de lignosulfonato (i) Mira 94 e (ii) TecMult 829 SPX, e dois a base de policarboxilato (i) ADVA 525 e (ii) TecMult 970, todos da marca Grace Construction Products. Os nanotubos de carbono analisados são os de paredes múltiplas, com comprimento dos tubos estimados entre $5 \mu \mathrm{m}$ e $30 \mu \mathrm{m}, 99 \%$ dos tubos com diâmetro externo entre $10 \mathrm{~nm}$ e $50 \mathrm{~nm}$, e pureza maior que 93\%, produzidos no Laboratório de Nanomateriais do Departamento de Física da UFMG. O cimento foi o de alta resistência inicial CPV-ARI Fácil da marca Holcim. Também foi utilizado um agente desincorporador de ar, o Densil 10, da marca Grace Construction Products. 


\subsection{Análise de dispersão dos NTCs em meio aquoso}

A análise da dispersão dos NTCs em meio aquoso foi feita por meio do granulômetro a laser da marca CILAS. No recipiente do granulômetro foi acrescentado de água, 40mg de NTC e $4 \mathrm{ml}$ para cada um dos quatro dispersantes, separadamente. Essa proporção permitiu a obscuração exigida pelo equipamento (entre 10 e 20). Dessa forma, com o equipamento foi realizada a leitura granulométrica e o registro dos resultados para as quatro soluções, com as proporções supracitadas.

\subsection{Preparação e caracterização dos nanocompósitos}

Foram elaborados nanocompósitos de cimento com nanotubos de carbono dispersos nos aditivos que apresentaram resultados de granulometria mais discrepante (ADVA 525 e Mira 94). Os NTCs foram misturados em proporções distintas em relação ao peso do cimento (Referência com $0 \%, 0,15 \%, 0,30 \%$ e $0,50 \%$ ), e sonificados pelo período de 2 horas.

Para a determinação do fator água/cimento $(\mathrm{a} / \mathrm{c})$ foram conduzidos ensaios a fim de verificar a consistência das pastas, de modo que ambas as referências tivessem mesma proporção de plastificante em relação ao peso total de cimento e trabalhabilidade similar. A relação $\mathrm{a} / \mathrm{c}$ adotada foi de $\mathrm{a} / \mathrm{c}=0,275$ para $\mathrm{o}$ policarboxilato, e $\mathrm{a} / \mathrm{c}=0,30$ para o lignosulfonato, e a adição de surfactante foi na proporção de $0,45 \%$ em relação ao peso do cimento. Foram adotados diferentes fatores a/c devido a potencialidade de cada plastificante. O ADVA 525, a base de policarboxilato, é considerado um aditivo superplastificante, no qual, considerando uma pasta de mesma consistência, permite maior redução da água de amassamento se comparado ao Mira 94, plastificante a base de lignosulfonato.

Imediatamente após a preparação das pastas de cimento, as mesmas foram ensaiadas quanto a consistência. Os aparelhos utilizados para a realização desse ensaio encontram-se descritos na norma NBR NM 43 - Cimento Portland - Determinação da pasta de consistência normal (ABNT, 2003). A sonda do aparelho de Vicat foi estacionada na superfície da pasta com auxílio de um parafuso e em seguida desparafusada. A nova posição da sonda em relação a placa base após 30 segundos foi determinada e este valor foi registrado para análise da consistência.

A mesma pasta preparada para determinação da consistência foi utilizada para o preenchimento de 16 (dezesseis) moldes cilíndricos de $05 \mathrm{~cm}$ de diâmetro e $10 \mathrm{~cm}$ de altura, originando corpos de prova (CP), no qual 04 (quatro) foram destinados à ensaios de compressão axial a 7 dias, 04 (quatro) à ensaios de tração por compressão diametral a 7 dias, 04 (quatro) à ensaios de compressão axial a 28 dias e 04 (quatro) à ensaios de tração por compressão diametral a 28 dias.

Após a preparação dos CPs, eles foram mantidos a temperatura ambiente por 24 horas, desmoldados, e em sequência foram mantidos em tanque com água saturada com cal pelo período de 7 e 28 dias. Completado tais idades, os corpos de prova a serem submetidos ao ensaio de compressão foram retificados, no equipamento Servocut 301-mm-Abrasive Cutter da marca Metkon, a fim de obter uma superfície regular para distribuição uniforme da tensão. 
O Ensaio foi realizado em um equipamento universal da marca EMIC com célula de carga de $300 \mathrm{kN}$ para compressão axial e $20 \mathrm{kN}$ para compressão diametral. A velocidade do ensaio foi de $0,20 \mathrm{MPa} / \mathrm{seg}$ e $1 \mathrm{~mm} / \mathrm{min}$ para compressão axial e diametral, respectivamente. Para o ensaio de tração, foi utilizado um aparato desenvolvido especialmente para a realização desse ensaio, conforme ilustra a Figura 01.

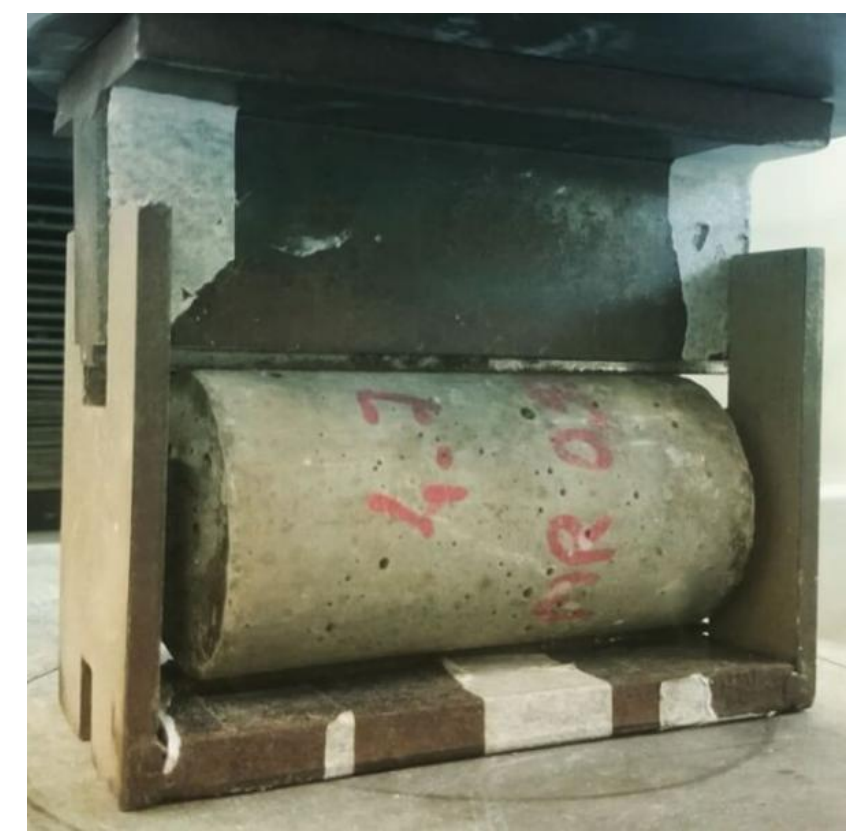

Figura 01 - Aparato desenvolvido para ensaio de tração por compressão diâmetral.

\section{DISCUSSÃO E RESULTADOS}

A Tabela 01 e a Figura 02 indicadas abaixo representam os resultados obtidos pelo granulômetro a laser, indicando o diâmetro em $\mu \mathrm{m}$ do peso de NTC passante.

Tabela 01 - Diâmetro das partículas de NTCs dispersos em surfactantes.

\begin{tabular}{ccccc} 
Aditivo & Base Química & $\begin{array}{c}\text { Diâmetro } \\
\text { a } 10 \%\end{array}$ & $\begin{array}{c}\text { Diâmetro } \\
\text { a } 50 \%\end{array}$ & $\begin{array}{c}\text { Diâmetro } \\
\text { a 90\% }\end{array}$ \\
\hline Mira 94 & Lignosulfonato & 45,734 & 124,486 & 380,477 \\
\hline Tecmult 829 SPX & Lignosulfonato & 61,629 & 203,580 & 426,783 \\
\hline ADVA 525 & Policarboxilato & 66,617 & 222,72 & 433,217 \\
\hline Tecmult 970 & Policarboxilato & 61,511 & 204,509 & 427,313 \\
\hline
\end{tabular}




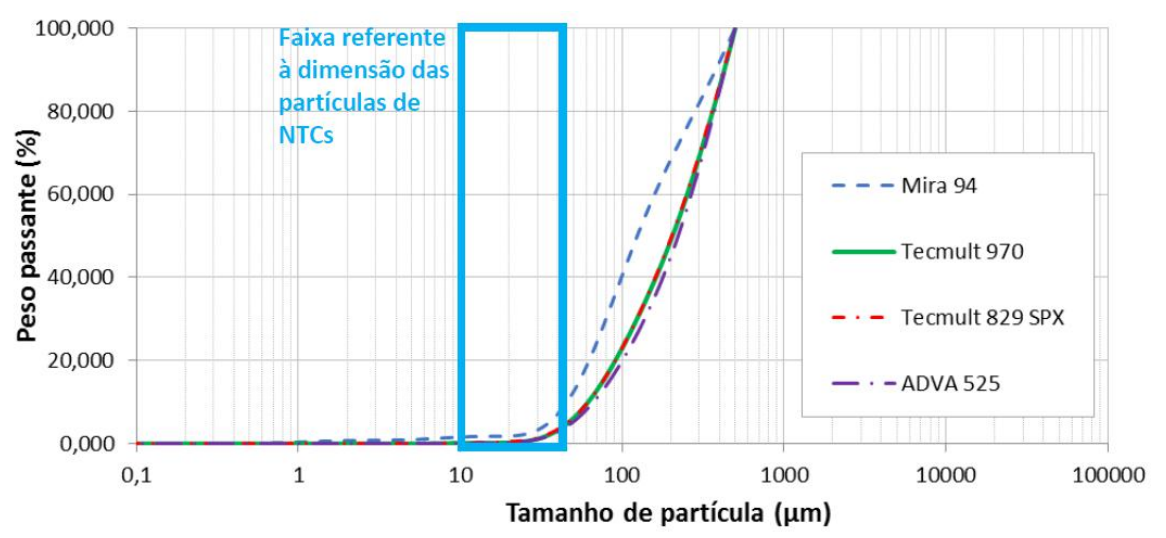

Figura 02 - Curva Granulométrica indicando a dispersão dos NTCs em surfactantes.

Como os ensaios foram realizados com uma mesma amostragem de NTCs, a diferença de granulometria apontada é em função da tendência do nanomaterial em se aglomerar na presença da água. Os resultados sugerem que o Mira 94 representa uma melhor dispersão já que indicou a menor granulometria das partículas de NTCs. O Tecmult 829SPX apresentou resultados similares ao Tecmult 970, sugerindo que ambos apresentem características similares para induzir a dispersão dos NTCs, apesar de serem de bases químicas diferentes. Pode ser sugerido que a dispersão dos NTCs em presença do Adva 525 permitiu maior aglomeração de nanomaterial e como consequência registrou partículas de maiores dimensões.

Os resultados da caracterização dos compósitos obtidos quanto a consistência encontram-se descritos na Tabela 02. As pastas de cimento foram preparadas utilizando NTCs dispersos em meio aos aditivos Mira 94 (a base de lignosulfonato) e ADVA 525 (a base de policarboxilato), que apresentaram os resultados mais discrepantes na análise granulométrica.

Tabela 02 - Distância do estacionamento da sonda em relação a placa base após 30 segundos.

\begin{tabular}{cc}
\hline Pasta de cimento & Consistência \\
\hline REF-MR & 24 \\
\hline $0,15-\mathrm{MR}$ & 25 \\
\hline $0,30-\mathrm{MR}$ & 31 \\
\hline $0,50-\mathrm{MR}$ & 33 \\
\hline REF-AD & 25 \\
\hline $0,15-\mathrm{AD}$ & 28 \\
\hline $0,30-\mathrm{AD}$ & 33 \\
\hline $0,50-\mathrm{AD}$ & 35 \\
\hline
\end{tabular}


A presença dos NTCs nas pastas de cimento fez com que a sonda apresentasse dificuldade de deslocamento através da pasta e estacionasse a uma distância maior da placa base, o que sugere que quanto maior a proporção do nanomaterial presente na pasta de cimento, menor a trabalhabilidade apresentada.

Os resultados da caracterização dos compósitos obtidos quanto nos ensaios de compressão e de tração por compressão diametral estão indicados na Tabelas 03 e nas Figuras 03 e 04:

Tabela 03 - Caracterização das pastas de cimento quanto a resistência à compressão e tração.

\begin{tabular}{ccccccc}
\hline $\begin{array}{c}\text { Pasta de } \\
\text { cimento }\end{array}$ & $\begin{array}{c}\text { Resistência } \\
\text { média a } \\
\text { compressão } \\
\text { (MPa) }\end{array}$ & $\begin{array}{c}\text { Desvio } \\
\text { Padrão a } \\
\text { compressão } \\
(\mathrm{MPa})\end{array}$ & $\begin{array}{c}\text { Ganho de } \\
\text { resistência a } \\
\text { compressão } \\
(\%)\end{array}$ & $\begin{array}{c}\text { Resistência } \\
\text { média a } \\
\text { tração } \\
(\mathrm{MPa})\end{array}$ & $\begin{array}{c}\text { Desvio } \\
\text { Padrão } \\
\text { a tração } \\
\text { (MPa) }\end{array}$ & $\begin{array}{c}\text { Ganho de } \\
\text { resistência } \\
\text { a tração } \\
(\%)\end{array}$ \\
\hline REF-MR-7 & 57,72 & 7,22 & - & 3,33 & 0,02 & - \\
\hline $0,15-M R-7$ & 56,56 & 0,07 & -2 & 2,68 & 0,03 & -20 \\
\hline $0,30-M R 7$ & 53,91 & 0,84 & -7 & 3,20 & 0,01 & -4 \\
\hline $0,50-M R-7$ & 55,00 & 5,92 & -5 & 2,98 & 0,01 & -11 \\
\hline REF-MR-28 & 63,82 & 6,49 & - & 2,78 & 0,91 & - \\
\hline $0,15-M R-28$ & 75,88 & 6,11 & 19 & 2,77 & 0,81 & 0 \\
\hline $0,30-M R-28$ & 67,07 & 11,21 & 5 & 2,91 & 0,22 & 5 \\
\hline $0,50-M R-28$ & 66,04 & 7,62 & 3 & 2,70 & 0,98 & -3 \\
\hline REF-AD-7 & 62,76 & 2,96 & - & 3,13 & 0,58 & - \\
\hline $0,15-A D-7$ & 60,58 & 2,08 & -3 & 3,18 & 0,66 & 2 \\
\hline $0,30-A D-7$ & 64,80 & 1,01 & 3 & 3,04 & 0,43 & -3 \\
\hline $0,50-A D-7$ & 63,45 & 5,76 & 1 & 3,66 & 0,49 & 16 \\
\hline REF-AD-28 & 68,51 & 9,86 & - & 3,74 & 0,73 & - \\
\hline $0,15-A D-28$ & 61,64 & 13,25 & -10 & 3,57 & 0,50 & -4 \\
\hline $0,30-A D-28$ & 65,26 & 13,85 & -5 & 3,12 & 0,76 & -17 \\
\hline $0,50-A D-28$ & 64,26 & 2,01 & -6 & 4,04 & 0,61 & 8 \\
\hline
\end{tabular}




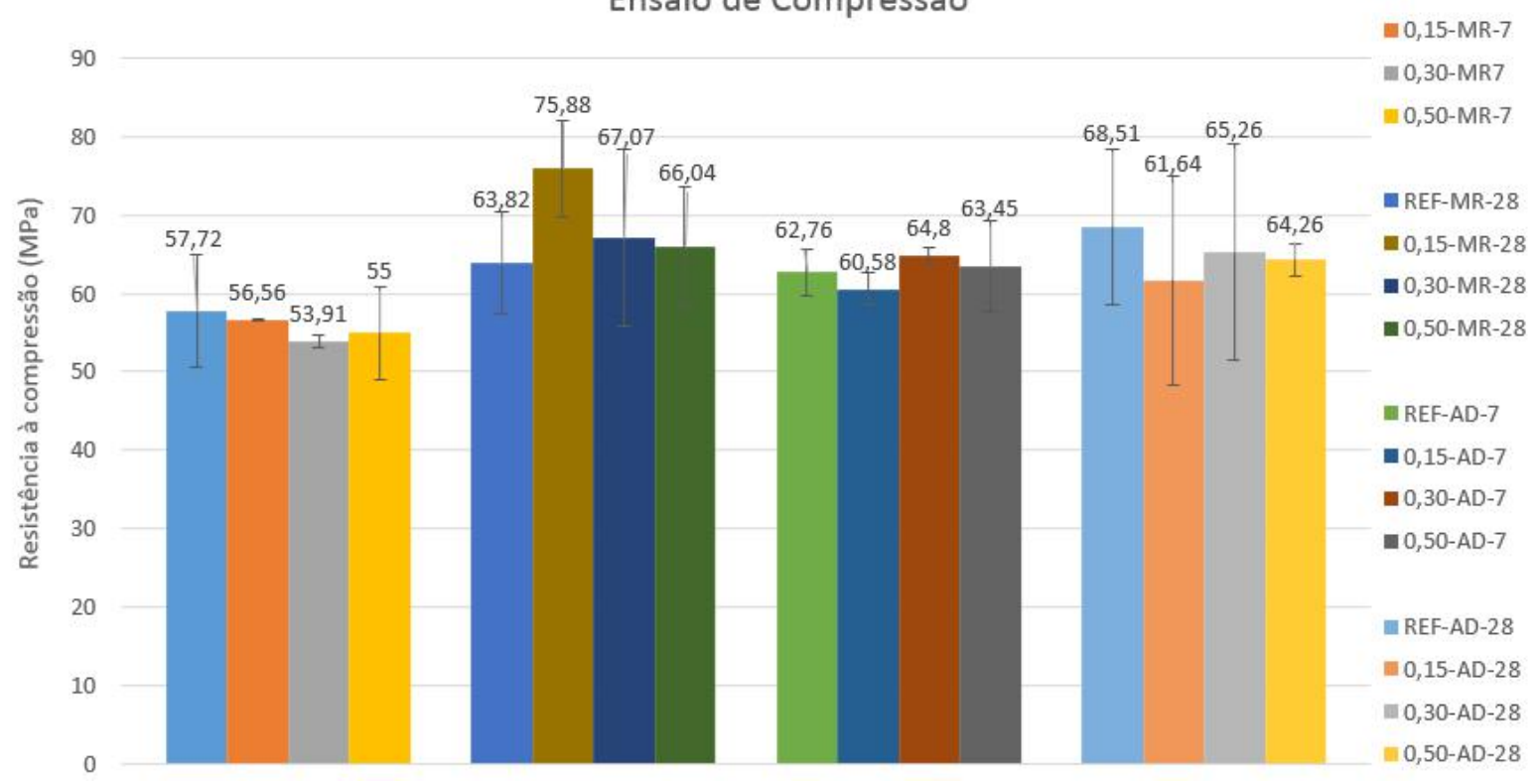

Figura 03 - Caracterização das pastas de cimento quanto a resistência à compressão.

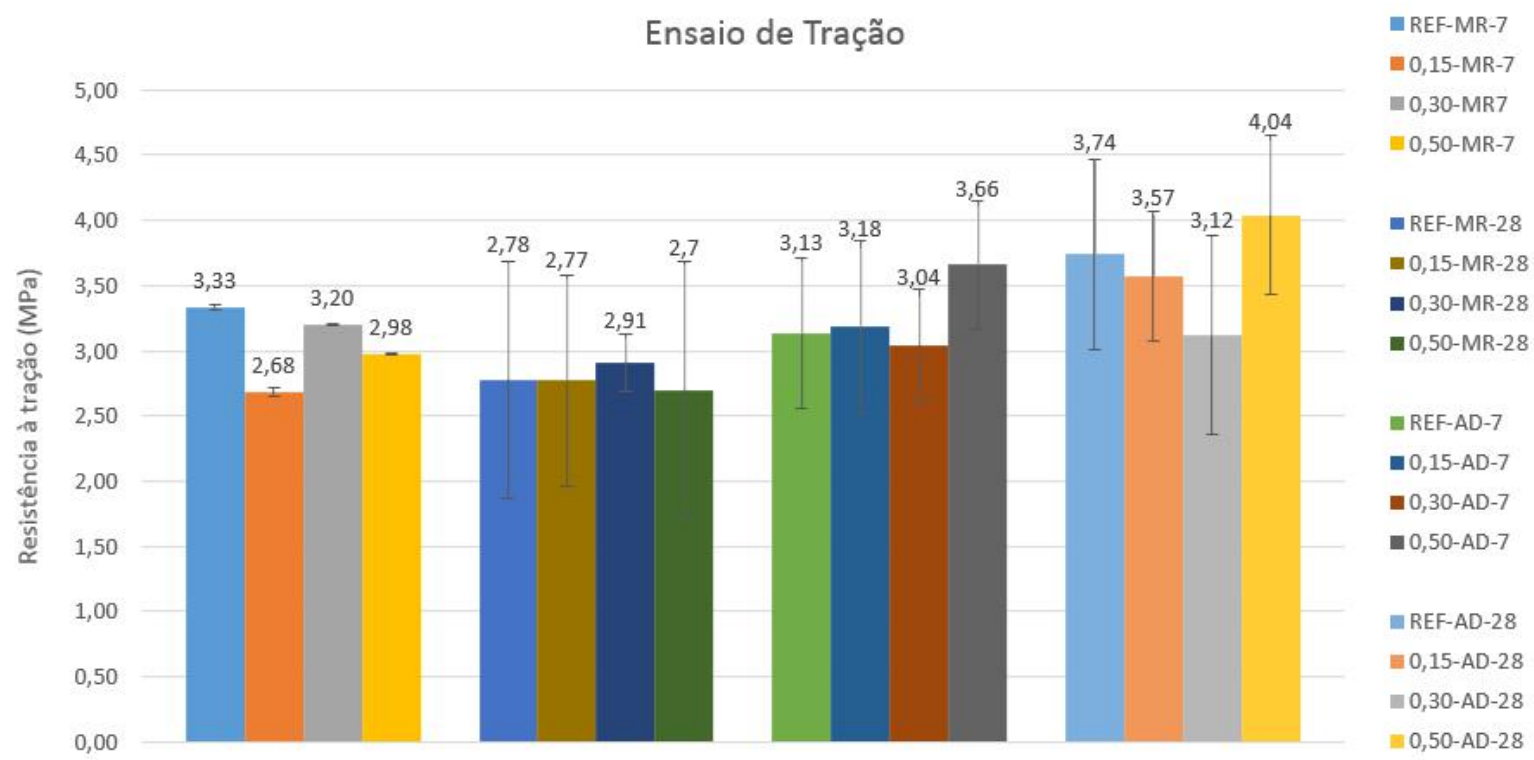

Figura 04- Caracterização das pastas de cimento quanto a resistência à tração.

Em presença do Adva 525, apesar de ter sido observado um ganho de $16 \%$ e $8 \%$ na resistência à tração com incorporação de 0,50\% de NTCs nas idades de 7 e 28 dias, respectivamente, os resultados das pastas de cimento em presença de NTCs foram, de modo geral, inferiores aos apresentados nas referências, para as duas idades analisadas. Na mesma proporção de $0,50 \%$ de NTCs as resistências à compressão não apresentaram ganhos, o que 
sugere dispersão pouco efetiva nas proporções adotadas para preparação de pastas de cimento. Esse resultado não vai ao encontro da conclusão obtida no trabalho de COLLINS et al. (2012), no qual os compósitos com $0,50 \%$ de NTCs dispersos no policarboxilato apresentaram melhorias nas propriedades. Essas diferenças, no entanto, podem ser explicada pelas diferenças nos métodos de dispersão e materiais adotados.

Com $0,15 \%$ de NTCs em presença do Mira 94, que é a base de lignosulfonato, obtevese ganho de $19 \%$ a 28 dias na resistência a compressão. Para resistência a tração, entretanto, o maior ganho foi de apenas 5\%, também a 28 dias, em presença de $0,30 \%$ do nanomaterial. Esses ganhos, no entanto, foram pontuais, e não podem ser apontados como indicativo ou tendência de ganhos de resistência associados à presença e dispersão efetiva dos NTCs.

\section{CONCLUSÃO}

Nas proporções adotadas, os quatro aditivos se mostraram eficientes na dispersão dos NTCs em meio aquoso. Os resultados do granuômetro indicam que o Mira 94, a base de lignosulfonato, aparenta uma melhor dispersão em meio aquoso, uma vez que permitiu a leitura de partículas menores. Já que a dispersão em Adva 525, a base de policarboxilado, permitiu maior aglomeração, fato associado ao registro de partículas de maiores dimensões.

Apesar dos resultados extremos serem de base química diferente, a similaridade do resultado apresentado pelo Tecmult 829SPX e Tecmult 970 sugere que as demais características intrínsecas dos surfactantes podem ser mais relevantes na dispersão que a base química o que pode ser apontado como justificativa para a diferença obtida nos resultados de COLLINS et al. (2012) e do presente trabalho.

Os experimentos confirmaram que a presença dos NTCs influencia na consistência das pastas de cimento: quanto maior o percentual de NTCs, menor a trabalhabilidade, corroborando o resultado apontado por COLLINS et al. (2012).

Apesar do ensaio por granulometria a laser ter sugerido boa dispersão em meio aquoso, a dispersão obtida por meio de surfactantes, para a preparação de nanocompósitos de cimento não se mostrou eficaz. As amostras ensaiadas em presenças dos NTCs não apresentaram ganhos significativos de melhorias na resistência em função da aglomeração do nanomaterial.

O uso de maiores proporções de plastificante poderia promover maior dispersão dos NTCs, no entanto, grandes proporções para preparação de compósitos de cimento não são recomendadas porque pode promover efeitos negativos, como incorporação de ar, retardamento de pega e inibição da hidratação. Para tentativa de melhores resultados, menores concentrações de NTCs podem ser adotadas, o que resultará numa menor relação NTCs:Surfactante.

\section{AGRADECIMENTOS}

Os autores agradecem à CAPES, ao CEFET-MG, ao CNPq e à FAPEMIG pelo auxilio no desenvolvimento do presente trabalho. 


\section{REFERÊNCIAS}

ABNT. NBR-43 - Cimento Portland - Determinação da pasta de consistência normal. Rio de Janeiro 2003.

COLLINS, F.; LAMBERT, J.; DUAN, W. H. The influences of admixtures on the dispersion, workability, and strength of carbon nanotube - OPC paste mixtures. Cement \& Concrete Composites, 2012.

FILHO, A. G. d. S.; FAGAN, S. B. Funcionalização de Nanotubos de Carbono. Química Nova, v. 30, 2007.

LIU, Y.; GAO, L.; SUN, J. Noncovalent Functionalization of Carbon Nanotubes with Sodium Lignosulfonate and Subsequent Quantum Dot Decoration. Journal of Physical Chemistry 2007.

MAKAR, J.; BEAUDOIN, J. Carbon nanotubes and their application in the construction industry. Special Publication-Royal Society Of Chemistry, v. 292, p. 331-342, 2004. ISSN 0260-6291.

MAKAR, J.; MARGESON, J.; LUH, J. Carbon nanotube/cement composites-early results and potential applications. 2005, CONFERENCE ON CONSTRUCTION MATERIALS.

MELO, V. S. et al. Macro-and Micro-Characterization of Mortars Produced with Carbon Nanotubes. ACI Materials Journal, v. 108, n. 3, 2011. ISSN 0889-325X.

PAULA, J. N. d. Comportamento reológico e mecânico de pastas de cimento fabricado com nanotubos de carbono crescidos em clínquer para poços de petróleo. Tese de doutorado, UFMG, 2014. 